\title{
Diagnosis and Surgical Treatment of Orbital Tumors of Various Localizations and Histostructures
}

\author{
OI Lystratenko ${ }^{1 *}$, AM Kardash ${ }^{2}$, DO Lystratenko ${ }^{3}$, AV Vashchenko ${ }^{4}$ and OF Smyrnova ${ }^{5}$ \\ ${ }^{1}$ Neurosurgeon of Donetsk Clinical Medical Association (DoKTMO), Ukraine \\ ${ }^{2}$ Head of the Department of Neurosurgery, Donetsk National Medical University (DoNNMU), Ukraine \\ ${ }^{3}$ Neurosurgeon of Donetsk Clinical Medical Association, Ukraine \\ ${ }^{4}$ Department of Neurosurgery, Neurosurgeon, Ukraine \\ ${ }^{5}$ Doctor of Medical Sciences, Ophthalmological clinic, Ukraine
}

*Corresponding author: OI Lystratenko, Neurosurgeon of Donetsk Clinical Medical Association (DoKTMO), Donetsk National Medical University, DoKTMO, Donetsk, Ukraine.

Received Date: January 27, 2020

Published Date: February 07, 2020

\section{Abstracts}

Introduction: The article reviews and analyzes the results of treatment of 32 patients with orbital tumors operated in the clinic, neurosurgery until the period from 2015 till September 2019.

Goals and objectives: coverage of clinical signs and symptoms, histology, diagnostic methods and treatment of patients operated on with orbital tumors during the period 2015-2019. Determination of optimal surgical approach to the tumors with different localization for performing the radical organ-preserving surgery, with maximal saving of visual functions, minimization of oculomotor disorders, patient disability.

Materials and methods: we analyzed the clinical cases of 32 patients undergoing treatment in DOKTMO with orbital tumors for the period from 2015 till September 2019.

Results: Patients were operated on with various approaches - transcutaneous, subconjunctive (without orbitotomy), fronto-orbito-zygomatic, pterional, subfrontal (neurosurgical approaches), transmaxillary, transethmoidal (ENT approaches). The choice of surgical approaches were individual. In 2 cases we become a backset of tumor growth: one patient with aggressive adenocarcinoma, after 18 months, leading to orbital exentration and the child, 9 years old with rhabdomyosarcoma after non-radical removal of tumors of subconjunctival approach. In all other cases, relapses were not detected, operation were organ-preserving oriented.

Conclusion: the results of treatment of patients with tumors directly depend on the choice of radicality tumor removal, which is associated with the choice of surgical approach, chemical, radiation treatment in postoperative period depending on the histological response. From our point of view, the most universal method for tumor removal with various localizations and sizes is fronto-orbito-zygomatic approach which provides the performance of radical organ-preserving operation with maximal vision saving, minimizing oculomotor disorders, disability of the patient, despite its technical complexity for ophthalmologist surgeons. In this regard, surgery of orbital tumors is subject to the competence of doctors of related specialties.

Keywords: Orbital tumor; Ptosis; Fronto-orbito-zygomatic approach; Subconjuctive approach; Rhabdomyosarcoma

\section{Introduction}

Tumors of the orbit is a common disease among ophthalmic pathology - up to $4 \%$. As it turned out, their diagnosis is a big problem not only for the ophthalmologist. Due to the anatomical proximity of the orbit to the cranial cavities and paranasal sinuses tumors of the orbit are very diverse, as they can arise from different anatomical structures of the orbit. In this regard not only oculists, but also ENT- doctors, maxillofacial surgeons and neurosurgeons can be involved in the diagnosis establishing and delivery of the surgical care of patients with orbital tumors.

The detection of orbital tumors in the Donetsk region since January 2015 has become much higher due to the use of the following examination algorithm: 
$>$ examination by an ophthalmologist, ENT specialist, neurologist;

$>\quad$ Ultrasound of the eye as a screening study;

$>$ CT scan with intravenous contrast, MRI to clarify the abundance of the process.

In the article we examined the results of the treatment of 32 patients operated on by us and performed the analysis of the surgical approaches depending on the localization and the nature of the process. We have proposed a scheme for determining the choice of surgical access, affecting on the radical removal of the tumor while maintaining all eye functions.

\section{Materials and Methods}

For the period from 2015 till September 2019, in Donetsk Neurosurgergical Clinic, we operated on 32 patients with tumors of the orbit of various localization and histostructure. Among the patients, 15 men (46.9\%) and 17 (53.1\%) women aged 2 to 79 years. Among them 7 (21.9\%) children - 3 boys and 4 girls.

The main symptoms of orbital tumors are: unilateral ptosis, exophthalm, diplopia, progressive decrease in vision in one eye, eye pain.

All patients were examined according to the algorithm above.

According to histological analysis, we distributed the clinical cases in the table (Table 1):

Table 1:

\begin{tabular}{|c|c|c|}
\hline Orbit tumors & Amount & Total \\
\hline Neuroblastoma & 1 & 32 \\
\hline Dermoid cyst & 1 & \\
\hline
\end{tabular}

\begin{tabular}{|c|l|l|}
\hline Fibromyoma & 3 & \\
\hline Meningioma & 2 & \\
\hline Osteosarcoma & 2 & \\
\hline Osteoma & 4 & \\
\hline Non-Hodgkin lymphoma & 1 & \\
\hline Lymphoma & 2 & \\
\hline Rhabdomyosarcoma & 2 & \\
\hline Angiofibroma & 1 & \\
\hline Plasmacytoma & 1 & \\
\hline Carcinoma & 3 & \\
\hline Adenocarcinoma & 1 & \\
\hline Fibrotic dysplasia & 1 & \\
\hline Wegener's granulomatosis & 1 & \\
\hline Basalioma & 1 & \\
\hline Hemangiopericytoma & 1 & \\
\hline Hemangioma & 2 & \\
\hline Filariasis & 1 & \\
\hline
\end{tabular}

Orbital tumors are various according location:

○ associated with the apical part of the orbit;

○ not associated with the apical part of the orbit;

- with intracranial growth,

○ with growth in the paranasal sinuses.

Depending on the location of the tumor, different surgical approaches should be preferred, which is displayed in a pie chart (Diagram 1):

Diagram :

We pay special attention to the selection of the optimal surgical approach to minimize the damage of the orbit structures during performance of organ-sparing surgery.

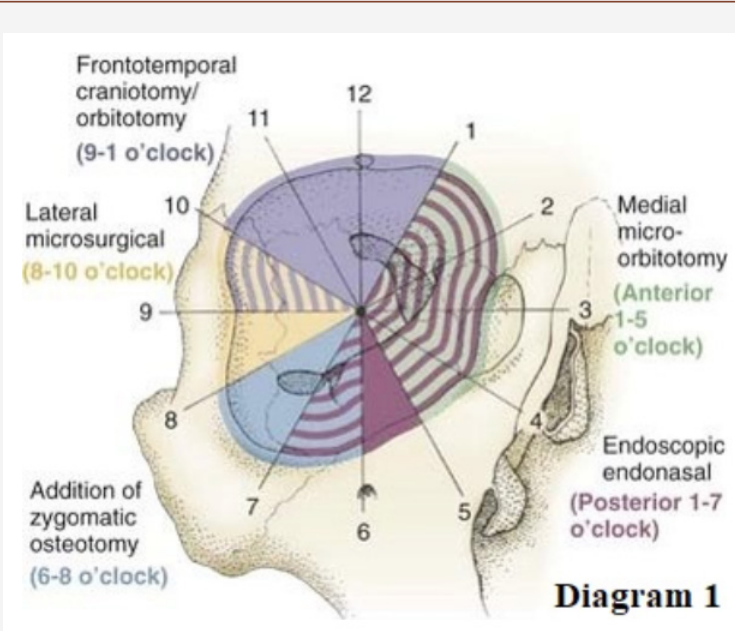

Diagram 1 
$>\quad$-lateral orbitotomy,

$>\quad$-transmaxillary orbitotomy,

$>\quad$-transetmoidal and transsphenoidal orbitotomy.

II. transcranial approaches:

$>$-frontal-orbital and subfrontal,

$>$-fronto-orbit-zygomatic, -pterional.

III. approaches without orbitotomy:

$>\quad$-transcutaneous

$>\quad$-transconjuctive.

Transcutaneous tumor removal, without orbitotomy, is the most often used by us when the tumor is located on the lower and lower medial edge of the orbit. The most often these are vascular tumors. We must not forget about filariasis, which can be regarded as a tumor of the orbit.

Clinical case. Woman 64 years old. The disease was taking place about 3 months when a painful swelling appeared on the right lower eyelid. Recurrent course with periodic edema, redness of the lower eyelid. On CT - soft tissue formation along the lower edge of the orbit. Intraoperatively was detected helminth in a fibrous capsule (Figure 1).

\section{Results}

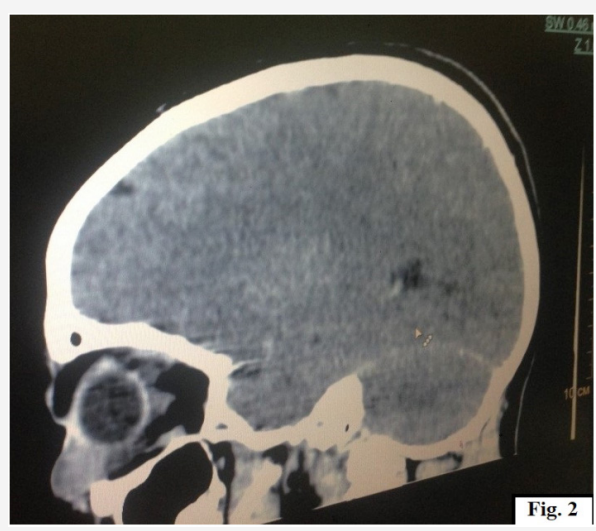

Figure 2: The child is 10 years old. Cavernous angioma. CT scan before operation.

Clinical case (Figure 2,3 \& 4). Child 10 years old. The diagnosis is cavernous angioma of the lower edge of the left orbit. The main complaint is pain in the left eye during its movement, limitation of the eyeball mobility. There was performed the operation transcutaneous removal of the tumor. The histological response is cavernous angioma (Figure 2,3 \& 4).

Lateral orbitotomy is used in a limited number of cases, more often when the tumor process is in the lateral, upper lateral region of the orbit, it is a minimally invasive orbitotomy. However, it requires fixation of the bone flap with titanium bridges. It is performed by cantotomy.

Clinical case (Figure 5,6). The patient is 57 years old. The diagnosis is plasmacytoma with damage of the frontal bone, upper lateral walls of the orbit. The main complaint is the restriction of the

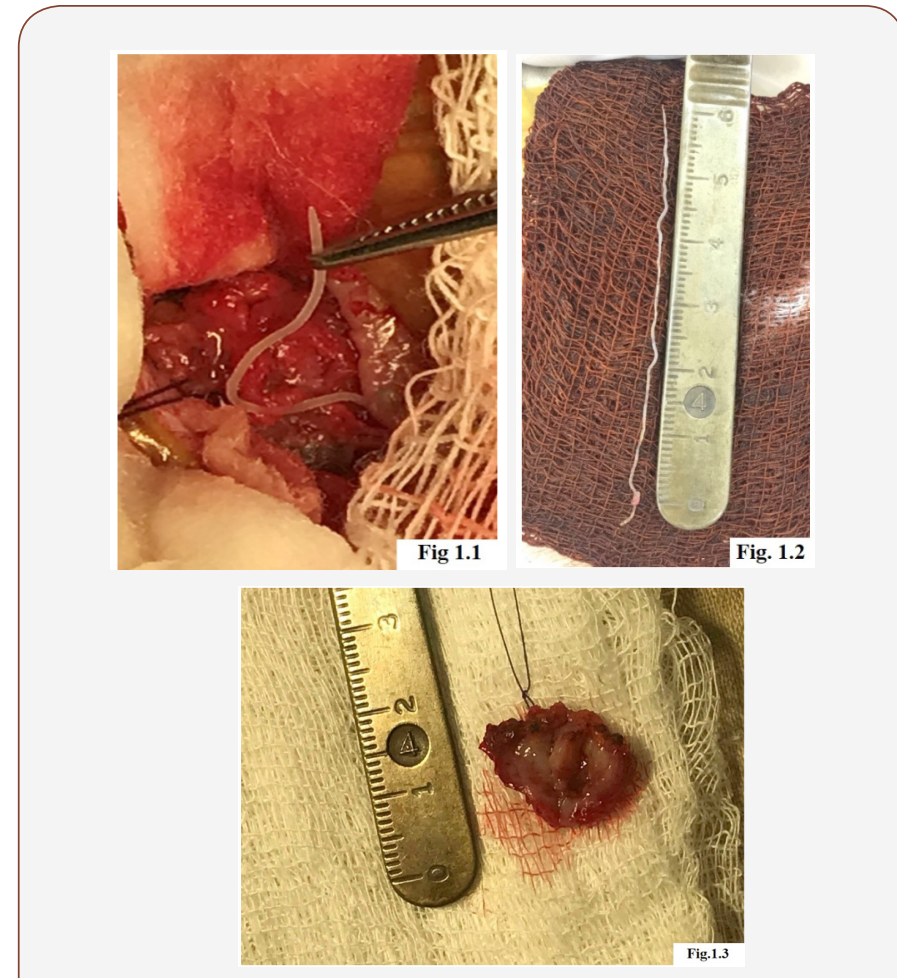

Figure 1: Photo. Filaria, fibrous capsule (intraoperative picture). eyeball mobility and pain in the eye. The operation was performed - lateral orbitotomy with resection of the portion of the lateral wall of the orbit and the part of the frontal bone affected by the tumor. After the operation the pain syndrome regressed and the mobility of the eyeball was restored.

The histological response is plasmacytoma. She underwent specific treatment with an oncohematologist (Figure 5,6).

Subconjunctival approach by cantotomy. It is a less traumatic modern approach, without orbitotomy. It is used to remove the orbital tumors of the lower medial and lower lateral location. Not effective for the location of the orbital tumors in the area of the intramuscular funnel, can be used for open biopsy of a diffuse tumor. 


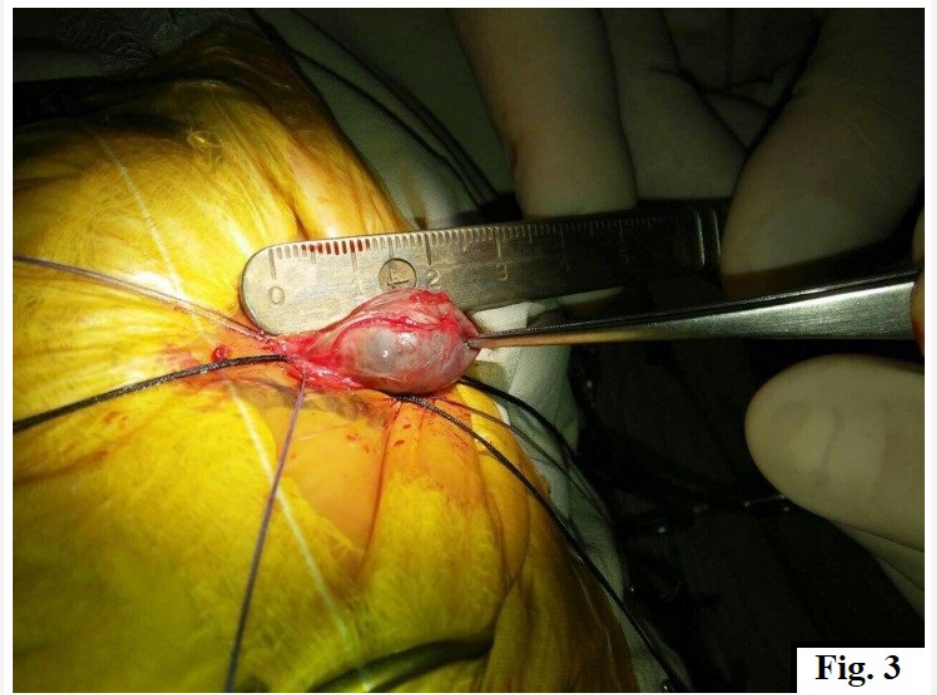

Figure 3: Transcutaneous removal of the orbit tumor (intraoperative picture).

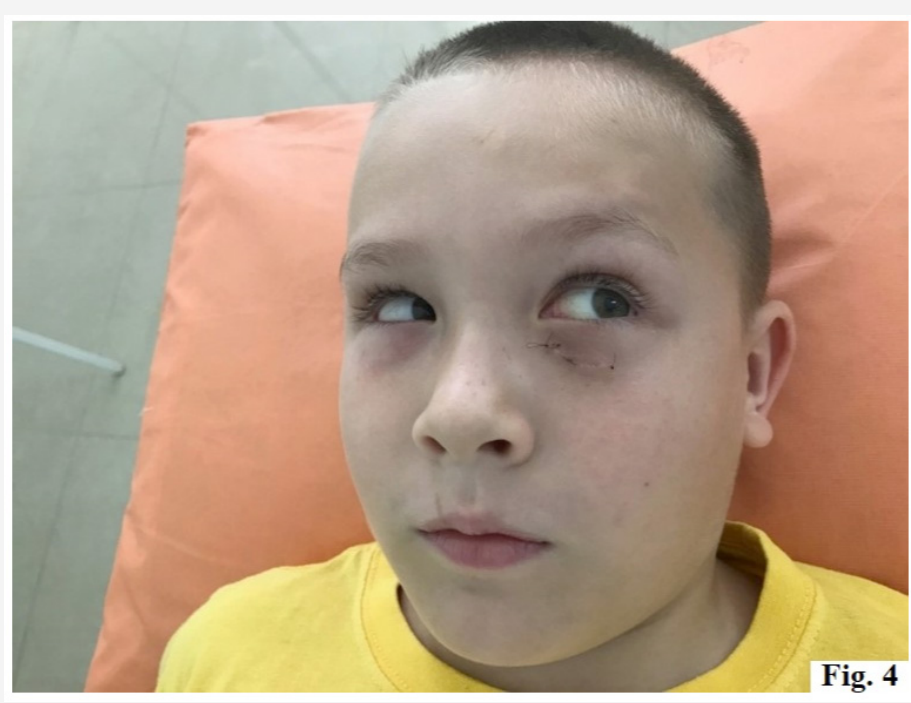

Figure 4: After the operation. Restored movement of the eyeball, regression of pain syndrome.

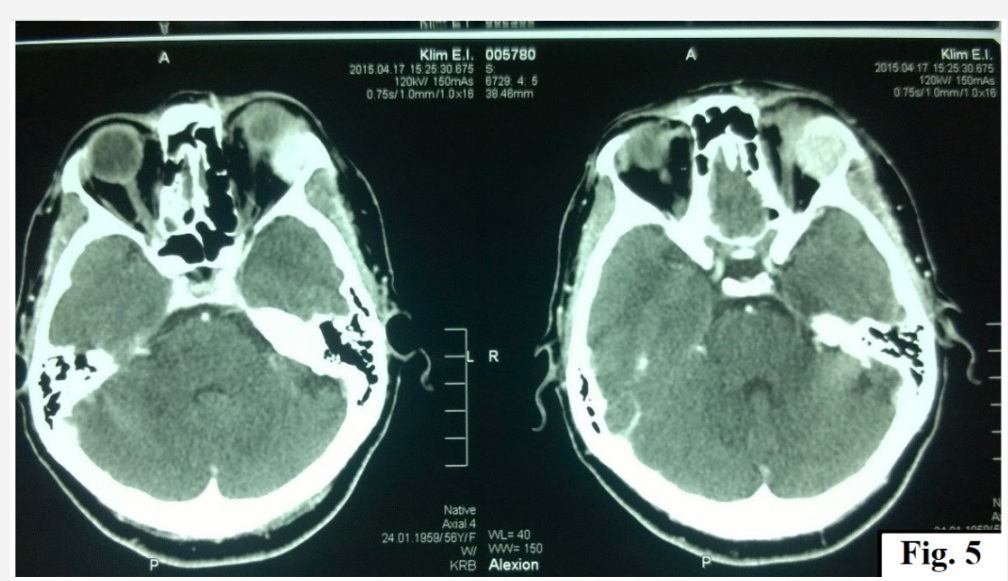

Figure 5: CT scan before operation. 


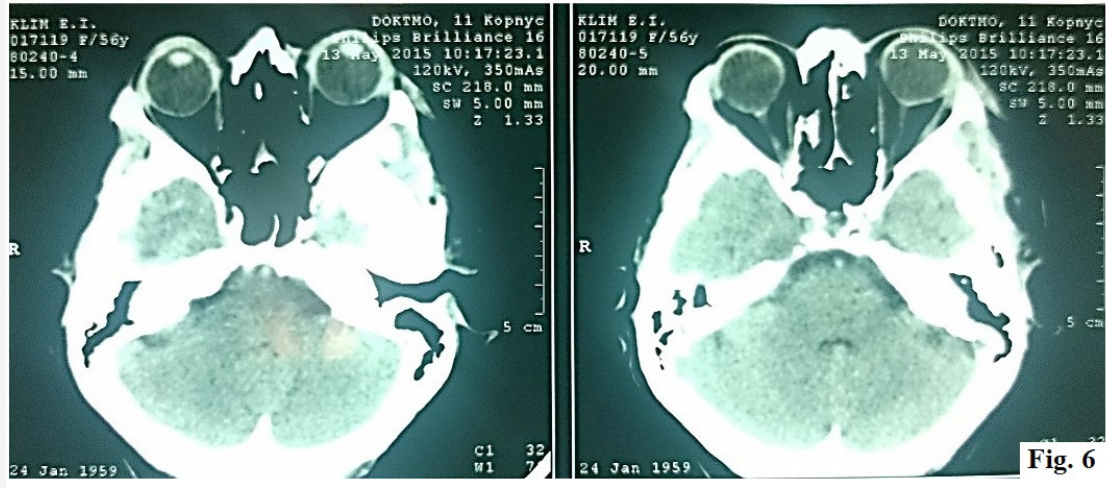

Figure 6: CT scan after operation.

Clinical case (Figure 7,8,9). This child is 6 years old. The girl was operated on in one of the ophthalmological clinics of Ukraine for a tumor of the left orbit with subconjunctival access. The histological response is rhabdomyosarcoma (Figure 7. Photo -15 days after operation). Due to lightning-fast relapse, severe pain, exophthalmos, progressive visual impairment, the child was reoperated in the neurosurgery clinic of DoKTMO. In order to radically remove the tumor there was chosen the fronto-orbito-zygomatic approach. (Figure 8. CT scan on the left before operation, on the right - after). After the operation regress of exophthalmos and pain, movements of the eyeball are full. The histological response is rhabdomyosarcoma. The child received a course of chemotherapy (Figure 9. Photo after combined treatment on the 30th day). No metastasis in the internal organs (Figure 7,8,9).

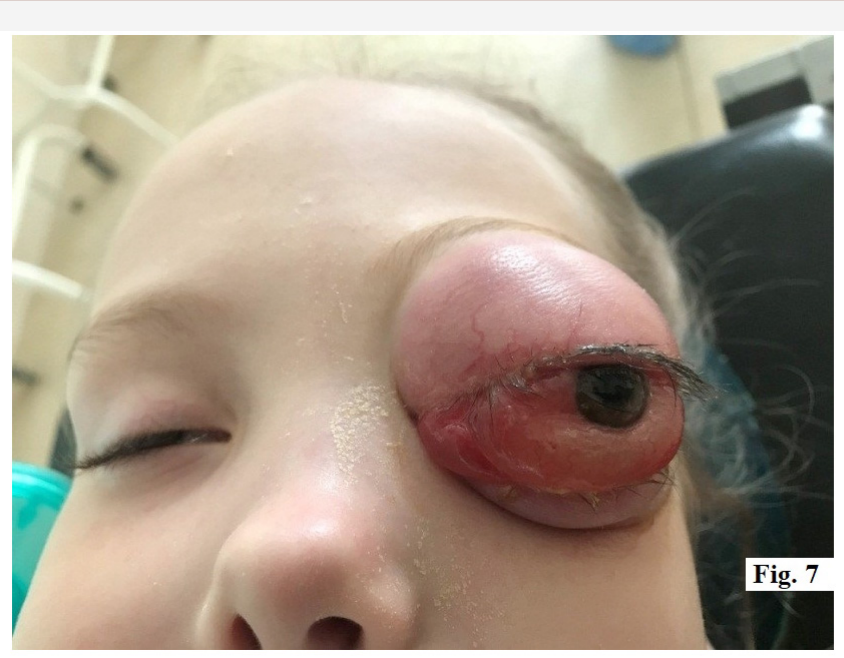

Figure 7: Photo -15 days after the 1st operation (subconjunctival approach, lightning-fast relapse).

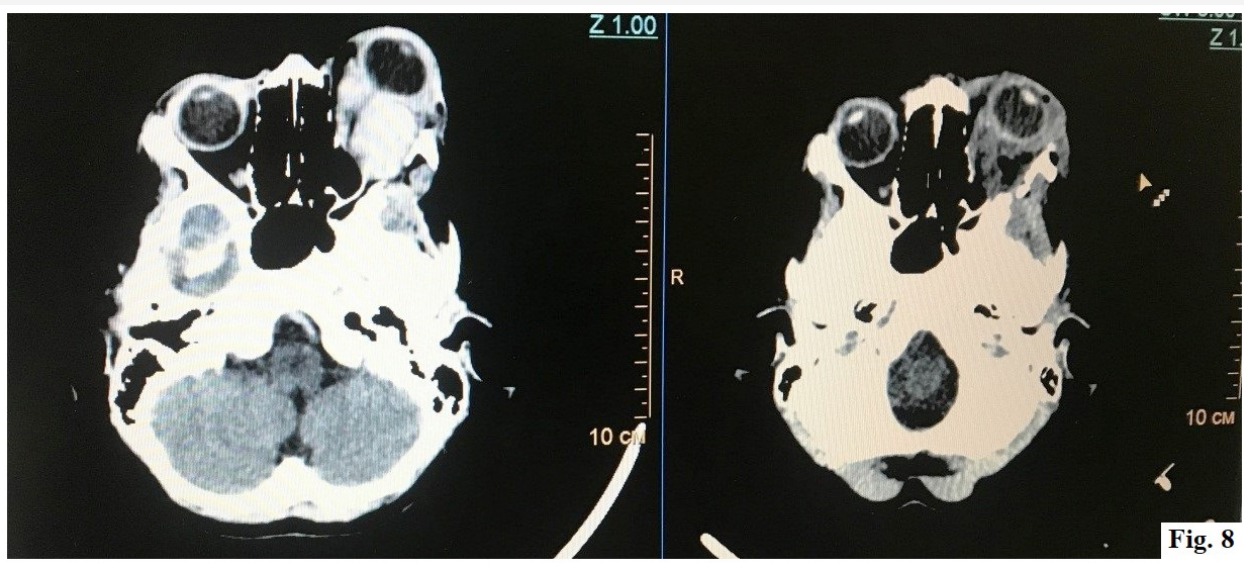

Figure 8:CT - Left before operation, right - after. 
Front-orbit-zygomatic access from our point of view is ideal, universal for removing orbital tumors with various localization: the optic nerve channel, intermuscular funnel, lacrimal gland tumors, lateral, lower lateral orbit regions, large diffuse tumors with intracranial spread.
Clinical case. Patient 62 years old. Diplopia, pain, exophthalmos, visual impairment. Symptoms increased in the indicated sequence within 1 year. Patient was operated on with fronto-orbito-zygomatic approach. In the postoperative period - regression of the described symptoms. The histological response is angiofibroma (Figure $10,11,12 \& 13)$

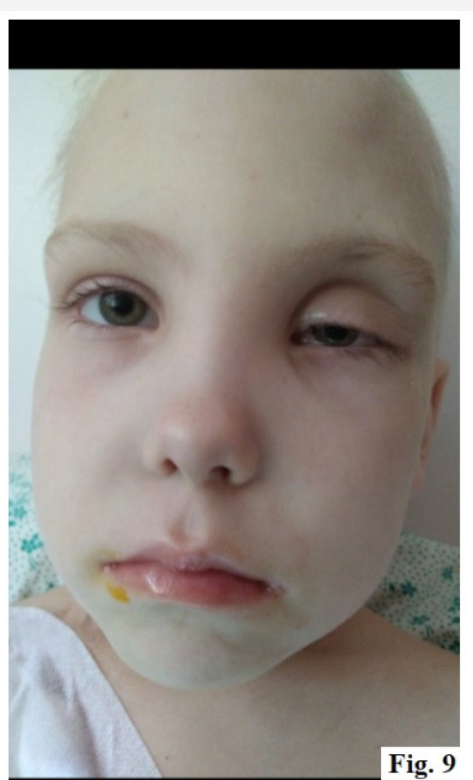

Figure 9:Photo after combined treatment on the 30th day. At the time of writing she is receiving further chemotherapy course.

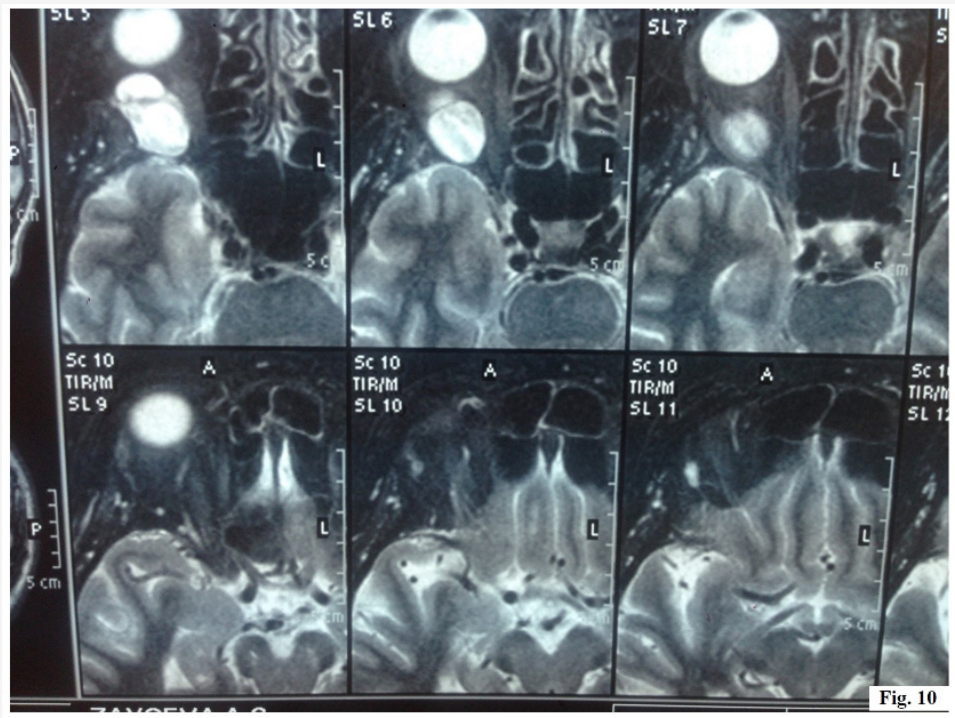

Figure 10: Tumor of the right orbit.

Separately, we provide the tactics of managing patients with tumors of the optic nerve. As a rule, these are patients of early childhood. In the treatment of such patients we were guided by literature data, the experience of foreign colleagues and a little of our own experience.

- $\quad$ Conservative treatment may be warranted because only $21 \%$ of these patients have tumor progression.
- $\quad$ Surgical treatment only by patients with progressive ptosis, blind eye, severe pain syndrome or when the expansion of the intracranial part of the optic nerve threatens chiasm, there exists fears of tumor expansion into the hypothalamus, 3rd ventricle. Fronto-orbito-zygomatic or pterional approach is used.

- Surgery includes resection of the optic nerve from the eyeball, within $2 \mathrm{~mm}$ of the chiasm. A puncture biopsy of the orbital 
component of the tumor is used to confirm the diagnosis if there occurs a progressive loss of vision or significant tumor growth.

- $\quad$ Radiation therapy with using of linear accelerator (50 Gy) is the treatment of choice for chiasmal gliomas.

- Radiation therapy does not affect the outcome of the disease, even when the tumor involves the hypothalamus and the third ventricle in the process (mortality rate: $28 \%$ ). You can become serious complications from the central nervous system.

\section{Here are a few clinical cases:}

-Clinical case (Figure 14) A child of 3 years. Lightning-fast development of the disease - pain, exophthalmos, vision loss, hypothalamic disorders.

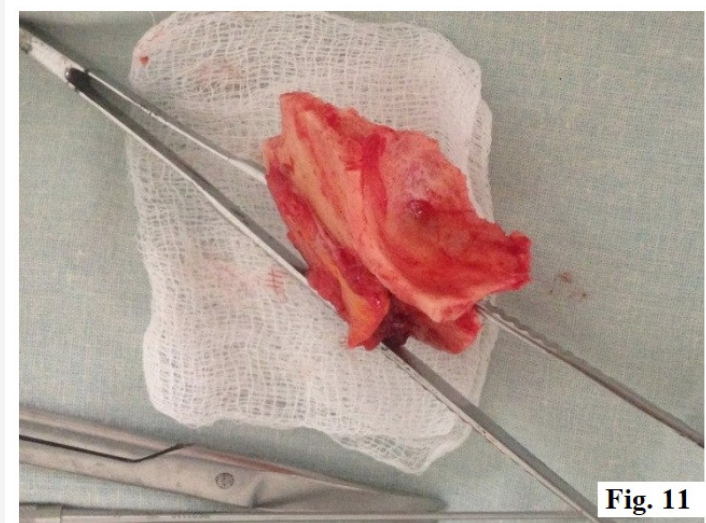

Figure 11: Fronto- orbito- zygomatic bone flap.

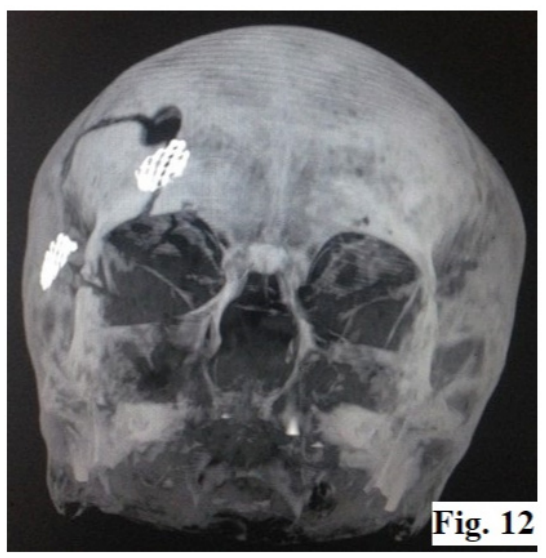

Figure 12: Fronto

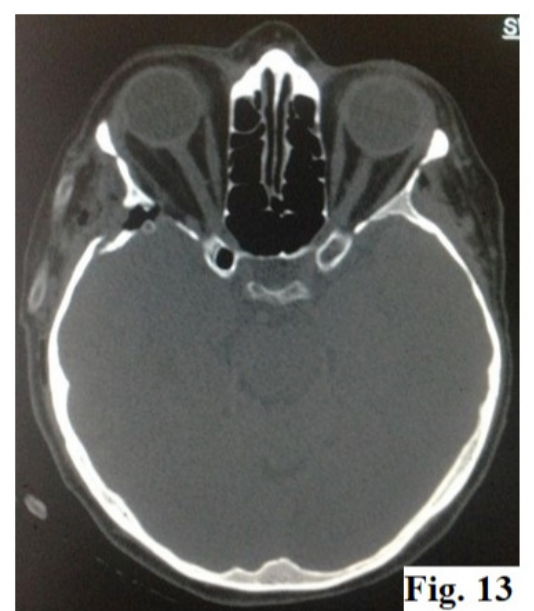

Figure 13: Tumor of the right optic nerve with the involvement of the eyeball, thalamic structures. 
An inoperable tumor of the right optic nerve with involving of the eyeball, chiasm and later the thalamic structures of the brain. A biopsy was not performed due to the severity of the child's condition - the transience of the process, the addition of hypothalamic disorders - most likely glioblastoma. Chemotherapy. The prognosis for life is not favorable (Figure 14).
Clinical case (Figure 15) A child of 8 months - there exists a glioma of the right optic nerve. The main complaint of parents is exophthalmos. Chemotherapy, positive dynamics. Currently, the child is 2 years 8 months old. Exophthalmos is regressed, vision on the affected eye is preserved. The child is under dynamic MRI supervision (Figure 15).

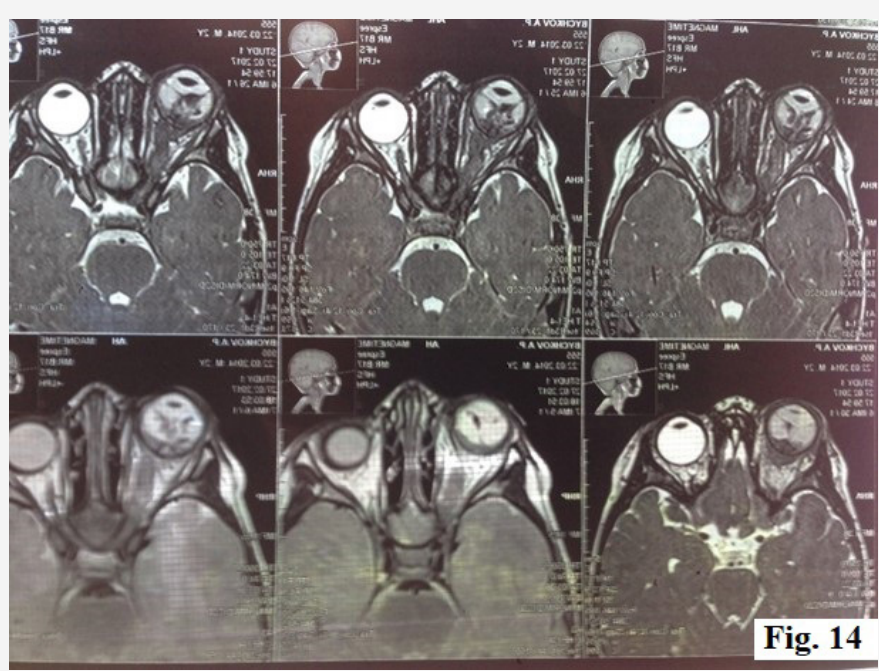

Figure 14: Tumor of the right optic nerve with the involvement of the eyeball, thalamic structures.

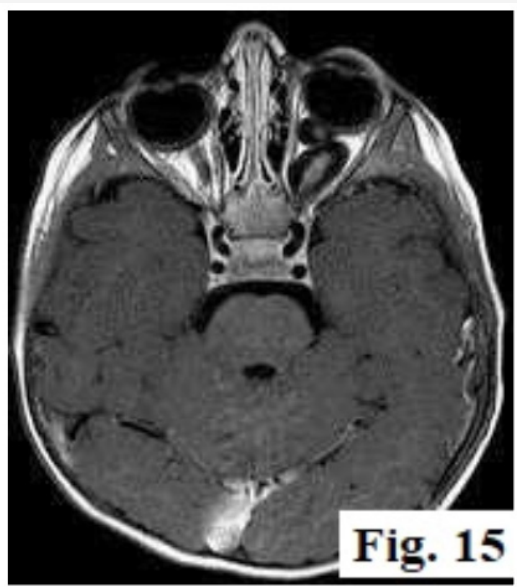

Figure 15: Child 8 months - glioma of the right optic nerve.

\section{Conclusions}

1. Tumors of the orbit are common diseases. The main symptoms of the orbital tumors are- unilateral ptosis, exophthalmos, diplopia, pain syndrome, progressive visual impairment. With the exclusion of endocrine pathology, a diagnostic algorithm should be performed to identify or exclude the tumors:

- Ultrasound of the eye, as a screening study;

- CT with intravenous contrast, MRI, to clarify the prevalence of the process;

○ ophthalmological examination, ENT specialist, neurologist.
2. The results of the treatment of patients with orbital tumors directly depend on the radicalism of the neoplasm removal, which is associated with the choice of surgical approach, the use of chemo-, radiation therapy in the postoperative period, depending on the histological response. In our opinion, the most universal method for removing orbital tumors of various localization and sizes is fronto-orbito-zygomatic approach which provides the implementation of radical organ-preserving surgery, with the maximum preservation of visual function, minimizing oculomotor disturbances, and patient disability, despite its technical complexity for surgeons ophthalmologists. In this regard, surgery of orbital tumors is a subject to the competence of doctors of related specialties. 


\section{Acknowledgement}

None.

\section{Conflict of Interest}

No conflict of interest.

\section{References}

1. De Groot AC (2000) Dermatological drugs, topical agents and cosmetics. In: Dukes MNG, Aronson JK, (Eds.), Meyler's side effects of drugs $\left(14^{\text {th }}\right.$ Edn.), Amsterdam: Elsevier: 449-452.

2. Marroni M, Bellomo G, Bucaneve G, Stagni G, Baldelli F (1993) Isotretinoin:possible cause of acute seizure and confusion. Ann Pharmacother 27: 793-794.

3. Springuel P, Roy G Isotretinoin (2006) (Accutane): myocardial infarction,cerebrovascular and thromboembolic disorders. Can Adverse React Newslett 16: 3 .

4. DRUGDEX (2007) Greenwood Village, CO: Thomson Micromedex. Edition expires 12/.

5. Laroche ML, Marcian Montoro F, Merle L, Vallat JM (2007) Cerebral ischemia probably related to Isotretinoin. Ann Pharmacother 41: 10731076.

6. Pritchard J, Appleton R, Howard R, Hughes RAC (2004) Guillain-Barré syndrome seen in users of isotretinoin. BMJ 328: 1537.
7. Modan B, Wagener DK (1992) Some epidemiological aspects of stroke: mortality/morbidity trends, age, sex, race, socio-economic status. Stroke 23: $1230-1236$

8. Moeller KE, Touma SC (2003) Prolonged thrombocytopenia associated with isotretinoin. Ann Pharmacother 37: 1622-1624.

9. Labiris G, Katsanos A, Karapetsa M, Ioanna M, Chatzoulis D (2009) Association between isotretinoin use and central retinal vein occlusion in an adolescent with minor predisposition for thrombotic incidents: a case report. J Med Case Reports 3: 58.

10. Lee JY, Mak CP, Wang BJ, Chang WC (1992) Effects of retinoids on endothelial cell proliferation, prostacyclin production and platelet aggregation. J Dermatol Sci 03: 157-162.

11. Georgala S, Schulpis KH, Potouridou I, Papdogeorgaki H (1997) Effects of isotretinoin therapy on lipoprotein (a) serum levels. Int J Dermatol 36: 863-864.

12. DeRose JJ, Madigan J, Umana JP, Prystowsky JH, Nowygrod R, et al. (1999) Retinoic acid suppresses intimal hyperplasia and prevents vessel remodeling following arterial injury. Cardiovasc Surg 7: 633-639.

13. Gornicki A, Gutsze A (2001) In vivo and in vitro influence of etretinate on erythrocyte membrane fluidity. Eur J Pharmacol 423: 127-134.

14. Gornicki A (2006) Influence of the retinoid acitretin on erythrocyte microrheology in vitro. Int J Clin Pharmacol Ther 44: 648-654.

15. Naranjo CA, Busto U, Sellers EM, Sandor P, Ruiz I, et al. (1981) A method for estimating the probability of adverse drug reactions. Clin Pharmacol Ther 30: 239-245. 